Epidemiology

\section{Occupational exposures and non-Hodgkin lymphoma: where do we stand?}

\section{A Blair}

\section{Commentary on the paper by Mester et al (see page 17)}

$T^{\mathrm{h}}$ he incidence of non-Hodgkin lymphoma (NHL) has increased markedly over the past four decades in most countries. ${ }^{1}$ The HIV epidemic contributed to this increase, beginning in the 1980s, but the remainder is largely unexplained. The remaining increase does not appear to be due to diagnostic changes and it cannot be strictly due to genetic factors because gene frequencies do not change rapidly enough to cause such large changes in rates over such a short period of time. We must, therefore, look to changes in the level and/or distribution of environmental factors to explain these rate increases. Possible environmental risk factors include occupational and environmental chemicals, microbes, diet, physical inactivity, and other lifestyle factors.

Although occupational exposures may contribute to the development of NHL, they are unlikely to explain the worldwide rise in incidence because the increase occurs among men and women and in developed and developing countries. No group of occupational exposures is likely to affect all of these populations. More widely spread exposures among the general population, such as viruses, diet, lifestyle activities, and general environmental chemicals, are more likely candidates.

Nonetheless, although occupational exposures may affect a smaller proportion of the population than general environmental exposures, studies in the workplace have played an important role in the identifying and characterising environmental carcinogens in the past and should be able to also contribute to the understanding of the rise in NHL incidence. Industrial chemicals move from contained locations at the worksite to the general environment through routine emissions, accidents, inclusion in commercial products, and storage at waste sites. Because workers often experience higher and more prolonged levels of exposure than the general population, they have long served as sentinels for chemical hazards in the general population.
The article by Mester and colleagues ${ }^{2}$ is another attempt to use the workplace to develop aetiological clues and provide leads for future studies to identify new environmental risk factors for NHL. They found elevated risks for NHL associated with several occupations (farmers, glass formers, and construction workers) and industries (food, beverage, tobacco, paper, printing, metal, and chemical). Some of these groups have been linked with NHL in other studies and are suggestive of possible hazards including pesticides, metals, particulates, engine exhausts, dyes and pigments, and solvents. Despite many leads from this and other studies, the literature on occupational exposures and risk of NHL is inconsistent. No workplace exposures have been conclusively identified as causal factors. ${ }^{3}$

If occupational and environmental chemicals make an important contribution to the aetiology of NHL, clear identification of such factors in future investigations probably requires two components. The first is investigation of risks among diagnostic subcategories of NHL, as in the Mester and colleagues ${ }^{2}$ paper. This is critical because there is growing evidence that the different subtypes of NHL have different aetiologies. ${ }^{3}$ The distribution of subtypes also varies geographically in the United States $^{4}$ and probably elsewhere as well. In addition, new molecular techniques suggest that NHL may be even more varied than previously thought. ${ }^{5}$ Future investigations need to characterise subtypes as precisely as possible. This requires large numbers of cases for study. International consortia of NHL studies-that is, EPILYMPH and INTERLYMPH, can provide these numbers and are being used for this purpose.

Although large studies are necessary, the more important methodological need is in the area of exposure assessment. Accurate assessment of occupational and environmental exposures is essential, but exceedingly difficult. Characterisation of the type and level of past exposures is typically easier in the workplace, where job descriptions, production procedures, and monitoring data are often available, than in the non-work environment. However, even in the workplace under the best of circumstances, misclassification of exposures is probably substantial. We know that a small amount of nondifferential exposure misclassification can bias risk estimates sharply towards the null, ${ }^{6}$ and that exposure misclassification generally does not fall in the "small" range. Differential misclassification can be equally damaging, but is less predictable in direction. Teschke and colleagues ${ }^{7}$ concluded that exposure assessment techniques for case-control studies display a "disappointing performance" with regard to validity. Exposure assessment in cohort studies may be better, but considerable improvement is necessary to be able to identify small to moderate levels of risk.

Although studies like the one in this issue $^{2}$ help to identify promising areas for future research and should be encouraged, we are unlikely to make major headway in the identification of new chemical risk factors for NHL unless we substantially improve the quality of occupational and environmental exposure assessments. Scientists engaged in exposure evaluation are developing some creative approaches such as using modular questionnaires to obtain detailed information on jobs, combining work histories with personal monitoring data, and obtaining biological measures of exposure, but progress is slow. Substantial intellectual and resource investment by the scientific establishment will be necessary before major improvements in exposure assessment are likely. Collaborations among chemists, industrial hygienists, bioinstrumentation engineers, biological monitoring experts, survey technicians, and chemical fate and transfer specialists will be necessary to push exposure assessment forward. Something akin to the human genome effort is probably required. Molecular epidemiology can help identify new risk factors through the study of genetically susceptible populations, evaluation of early markers of disease, and characterisation of mechanistic pathways, but these approaches also require high quality exposure assessments. Without accurate and reliable exposure assessments, we will miss important gene-exposure interactions, undervalue the contribution of environmental effects, and overestimate the effects of genes. ${ }^{8}$ Improvements in exposure assessment are necessary if we are to effectively evaluate environmental factors 
responsible for changing patterns of diseases like NHL.

Occup Environ Med 2006;63:1-2. doi: 10.1136/oem.2005.023978

Correspondence to: $\operatorname{Dr}$ A Blair, National Cancer Institute, Executive Plaza South, Room 8118, Bethesda, MD 20892, USA; blaira@ mail.nih.gov

Funding: this research was support by the Intramural Research Program of the NIH (National Cancer Institute)

\section{Competing interests: none}

I performed this work as part of my employment with the US government and consequently copyright cannot be assigned. The BMJ Publishing Group Ltd, however, is free to use this material on a worldwide basis and to publish it in OEM.

\section{REFERENCES}

1 Hartge P, Devesa SS. Quantification of the impact of known risk factors on time trends in nonHodgkin's lymphoma incidence. Cancer Res 1992;52:5566s-5569s.

2 Mester B, Nieters A, Deeg E, et al. Occupation and malignant lymphoma: a population based case control study in Germany. Occup Environ Med 2006;63:17-26.

3 Chiu BCH, Weisenburger DD. An update of the epidemiology of non-Hodgkin's lymphoma. Clinical Lymphoma 2003;4:161-8.

\author{
4 Morton LM, Wang SS, Devesa SS, et al. \\ Lymphoma incidence patters by WHO \\ subtypes in the United States, 1992-2001. \\ Blood 8 September 2005 \\ (Epub ahead of print). \\ 5 Staudt LM. Molecular diagnosis of \\ hematologic cancers. N Engl J Med \\ 2003;348:1777-85. \\ 6 Checkoway H, Pearce N, Kriebel D. Research \\ methods in occupational epidemiology. New \\ York: Oxford University Press, 2004. \\ 7 Teschke K, Olshan AF, Daniels JL, et al. \\ Occupational exposure assessment in \\ case-control studies: opportunities for \\ improvement. Occup Environ Med \\ 2002;59:575-94. \\ 8 Vineis P. A self-fulfilling prophecy; are we \\ underestimating the role of the environment in \\ gene-environment interaction research? \\ Int J Epidemiol 2004;33:945-6.
}

\section{Physical activity, psychological complaints, and occupational health}

\section{B Evanoff}

\section{Commentary on the paper by Bernaards et al (see page 10)}

( ccupational health professionals and researchers are primarily concerned with examining and preventing the specific health effects of exposures to chemical and physical agents that are unique to the work environment. When looking beyond work related diseases to other diseases and broader health outcomes such as disability, absenteeism, and general health status, we must also consider the effects of factors outside of work, and the interaction of these factors with work exposures. The article by Bernaards and colleagues ${ }^{1}$ provides further evidence of the importance of taking a broader view of worker health and safety.

The study by Bernaards and colleagues used data from the Dutch SMASH study (Study on Musculoskeletal disorders, Absenteeism, Stress, and Health), a three year prospective cohort study of over 1700 Dutch workers. Baseline and annual questionnaire data on strenuous leisure time physical activity were used to predict four health outcomes: depression, emotional exhaustion, perceived general health status, and work absenteeism due to psychological complaints. The study found that strenuous leisure time physical activity was associated with a lower risk of depression, emotional exhaustion, and poor general health, as well as with a lower risk of work absenteeism due to psychological complaints. The beneficial effects of exercise were strongest in persons with sedentary jobs. These results join those of a few other longitudinal studies showing that higher levels of leisure time physical activity are associated with lower risks of future depression and depressive symptoms. $^{23}$

Surprisingly, this study found that strenuous physical activity at a frequency of once or twice a week was associated with lower risks of depression than exercise at three or more times per week. Unfortunately, the relatively small number of workers in the highest category of exercise frequency meant that this study did not have the statistical power to accurately determine the shape of the dose-response curve, which differed in the various analyses conducted for different health outcomes in their study. A larger study would be needed to determine whether the psychological and work enabling health benefits of exercise increase in a monotonic dose-response fashion, whether there is a plateau with decreasing incremental benefits at higher exercise levels, or whether there is truly a "Ushaped" curve with decreasing psychological benefits resulting from more exercise. Though this latter possibility seems unlikely, it is conceivable that people prone to depression reported exercising more, or that "overtraining" led to depressive symptoms in some subjects. Another surprising result, which was not discussed by the authors, was the increasing prevalence of current depressive symptoms during the study, from $9.1 \%$ of all subjects at baseline to $15.8 \%$ three years later. This is especially surprising because people with depression at baseline were more likely to drop out of the study.

Though this study was carefully performed, it nonetheless has some methodological limitations, shared by other studies in this area, that limit conclusions regarding causality. One such problem is potential bias because the exposure of interest is likely to be affected by the outcome of interest: people with poor general health or depression are less likely to exercise than those with good health or without depression. The one year lagging of exposure used by the authors reduces, but probably does not eliminate this potential bias. Similarly, the relation between exercise frequency and work absenteeism is likely to be confounded by health and emotional status. The small number of subjects in the highest exercise category limited the conclusions that could be drawn regarding the level of exercise that provided benefits. Restricting the analysis of sick leave to those with sick leave attributed to psychological disorders left few subjects for analysis, and was probably subject to under-reporting. Complementing the results of this paper is another publication from the SMASH study which showed that all-cause sickness absences, and the duration of these absences, was lower among workers who reported regular physical activity. ${ }^{4}$

Despite methodological limitations, this study shows an important relation between strenuous recreational physical activity and psychological health. The 